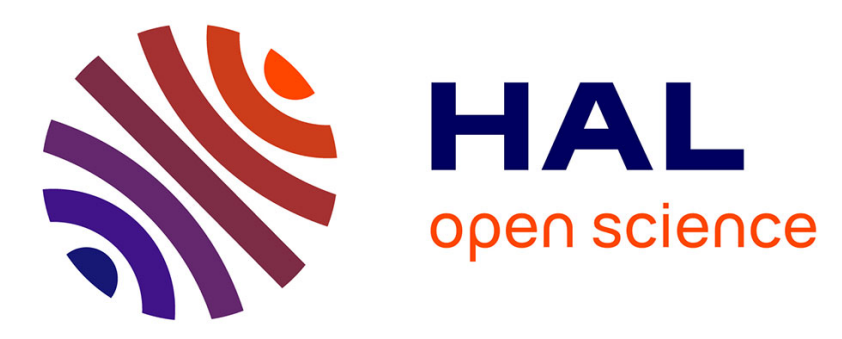

\title{
A reduced order observer for Switching-Mode Model state estimation
}

Hirsh Majid, Hassane Abouaïssa, Daniel Jolly, Gildas Morvan

\section{To cite this version:}

Hirsh Majid, Hassane Abouaïssa, Daniel Jolly, Gildas Morvan. A reduced order observer for SwitchingMode Model state estimation. 2013 International Conference on Control, Decision and Information Technologies (CoDIT), May 2013, Hammamet, Tunisia. pp.021-026, 10.1109/CoDIT.2013.6689513. hal-03353669

\section{HAL Id: hal-03353669 https://hal.science/hal-03353669}

Submitted on 27 Sep 2021

HAL is a multi-disciplinary open access archive for the deposit and dissemination of scientific research documents, whether they are published or not. The documents may come from teaching and research institutions in France or abroad, or from public or private research centers.
L'archive ouverte pluridisciplinaire HAL, est destinée au dépôt et à la diffusion de documents scientifiques de niveau recherche, publiés ou non, émanant des établissements d'enseignement et de recherche français ou étrangers, des laboratoires publics ou privés. 


\title{
A Reduced Order Observer for Switching-Mode Model State Estimation
}

\author{
Hirsh Majid, Hassane Abouaïssa, Daniel Jolly, Gildas Morvan
}

\begin{abstract}
In this paper, we investigate the application of a reduced-order observer for state estimation of freeway traffic flow described by the so-called Switching Mode Model (SMM). SMM is derived from the Cell Transmission Model and then applied to the reconstruction of the traffic density, considered as state variable. As the SMM is a hybrid system which switches depending on the traffic conditions along the freeway, the paper shows how, the proposed estimation method takes into account such different modes. Several numerical simulation with different data demonstrate the relevance of the proposed algorithm.

Index Terms-Reduced-order observer, traffic flow simulation, Switching mode model.
\end{abstract}

\section{INTRODUCTION}

Freeway traffic control and the development of intelligent transportation systems, which are able to provide continuous forecasting of the traffic and allow to alleviate the congestion phenomena, are closely related to the ability to have all needed information about the traffic state. Indeed the performance of any dynamic management action, usually requires the knowledge of both the present and the future traffic behaviour. Such information are provided by a set of sensors (detectors) which are already installed along the freeway infrastructure. The future traffic behaviour can be computed via a suitable traffic model. The most used sensors are the loop detectors, the video cameras, etc. Nevertheless, like any other device, the sensors are facing problems of cost, maintenance, and dysfunction. Moreover, loop detectors, for example could undermine the road surface (paved), and accelerates the deterioration of the infrastructure. Thus, reducing the number of sensors, can help to alleviate the costs of system. In this context, state estimation and reconstruction of missing data plays an important role.

The work proposed in this paper deals with traffic flow state estimation using a reduced order observer which was proposed by Luenberger [1].

The amount of attention devoted to the development of an estimation strategy using a traffic flow model has been relatively small compared to the modelling stage. Here we mention some works; Nahi [2] shows the ability to estimate the density closely in homogeneous situations. Various authors like Gazis and Knapp [3], and Knapp [4] have used Kalman Filtering techniques, they proposed a method which utilizes the time series of speed and flow data from each detector and

H. Majid (corresponding author), H. Abouaïssa, D. Jolly \& G. Morvan are with Univ. Lille Nord France, F-59000 Lille, France. U-Artois, LGI2A, EA. 3926 Technoparc Futura, F-62400 Béthune, France. hirshmajid@yahoo.com, (hassane. abouaissa, daniel.jolly, gildas.morvan)@univ-artois.fr generates crude estimates of vehicles count. These estimates are then filtered using a Kalman Filter. Extended Kalman Filters have also been utilized in traffic state estimation using second-order models [5]. Rashid R.Kohan [6] has designed a sliding mode state estimator for estimating state variables of traffic flow (speed, density) which is an estimator robust with respect to bounded modelling errors and disturbances. In 2003, Muñoz and al [7] derived a switching mode model (SMM) from the cell transmission model (CTM) which has been applied to the estimation of traffic densities at unmonitored locations along highway. In (2004) Sun and al [8], proposed an estimator using a mixture Kalman filtering (MKF) algorithm on the switching-mode traffic model, their estimator is able to provide estimated vehicle densities at unmeasured locations. In (2010) Staňková and al [9] dealt with the problem of the real time freeway traffic density estimation/prediction for a jump Markov linear model based on Daganzo's cell transmission model, to solve this problem they proposed a particle filteringbased estimation/prediction method. In 2011 Morārescu and al [10] proposed a new state estimation technique to reproduce the state variable (density) in the real time traffic forecasting by using switching mode model.

In this paper, an approach to estimate hybrid discretetime linear systems using Luenberger observer is presented. The proposed Luenberger observer for such kind of systems relies on the switching among different gains. Convergence conditions have been found to ensure the stability of error dynamics and the related gains may be selected by solving a set of linear matrix inequalities. The model used in the system is the switching mode traffic model (SMM). It is a macroscopic linear time-varying traffic flow model. It can be also defined as a hybrid system that switches among different sets of linear difference equations, or modes.

This paper is organized as follows:

- Section II, recalls the used macroscopic model.

- Section III, outlines the basis of the state reconstructor scheme used.

- in section IV, we apply the estimator on a section freeway. The section provides some numerical simulations and results.

- The conclusion, section V, summarizes the main gained results and lists some promising perspectives and further researches.

\section{TRAFFIC Flow Modelling}

Highway traffic flow system is an interesting but complex phenomenon to model. Many efforts have been made to 


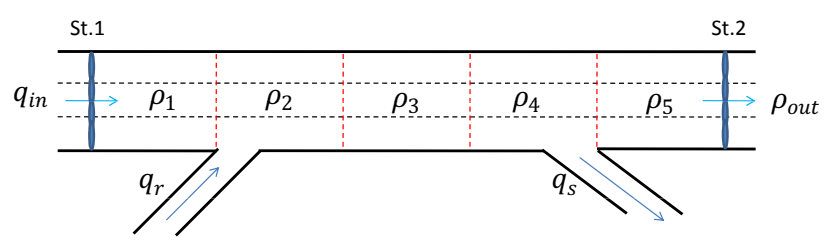

Figure 1. freeway Section

establish and validate models of highway traffic at different scales (microscopic, macroscopic). However, they are not easy to be used precisely for online estimation of the traffic states. Here, we are interesting on the macroscopic model. The first macroscopic model was developed by Lighthill and Whitham in 1955 [11] and Richard 1956 [12]. The model rests on the comparability of traffic flow on long crowded roads with flood movements in long rivers. Thus, the model is based on the equation of fluid dynamic, $(q=\rho v)$, where, $q$ is the traffic flow in vehicles/h. $\rho$, the traffic density in vehicles $/ \mathrm{km}$ and $(v)$, the mean speed in $\mathrm{km} / \mathrm{h}$ ), and on the equation of conservation law (see equation 1 ), in which $\rho(x, t)$ is the density of vehicles at point $x$ and at time $t$, $q(x, t)$ is the flow function defined on [0 and $\left.\rho_{m}\right]$, where $\rho_{m}$ is called jam density. This model suppose that traffic is in steady state.

$$
\frac{\partial \rho(x, t)}{\partial t}+\frac{\partial q(x, t)}{\partial x}=0
$$

The switching mode model is a macroscopic linear timevarying traffic flow model; it has been derived from a modified cell transmission model CTM [13], [14] that uses density instead of occupancy as its state variable and consider the triangular form of diagram fundamental. In the switching mode model, the cell transmission model is described as a hybrid system that switches between 5 sets of linear difference equations or modes (see table I), depending on the congestion status of the cells and the traffic situation of the mainline boundaries. At each time step, the densities and flows at boundary mainline segments are measured. There is at most one status transition (or wave front) [7] in the highway section. If both adjacent mainline segments are in the same traffic situation, i.e., both congested or free flow, so it is assumed that all mainline cells have the same traffic situation, either all congested, or all free flow. While, if the adjacent mainline segments are in different traffic situations, there exists a single wave front in one of the cells mainline, upstream of which all cells have congested (free flow) status, and downstream of which all cells have congested (free flow) status.

The highway segment divided into 5 cells shown in figure (1) is considered. The measured aggregate flows and densities at the upstream and downstream mainline detectors are denoted $q_{\text {in }}, \rho_{\text {in }}$ and $q_{\text {out }}, \rho_{\text {out }}$ respectively. All 5 modes of the SMM can be summarized as follows:

$$
\rho_{i}(t+1)=R_{i, s} \rho_{i}(t)+M_{i, s} u_{i}(t)+N_{i, s} \rho_{m, i}+O_{i, s} Q_{m, i}
$$

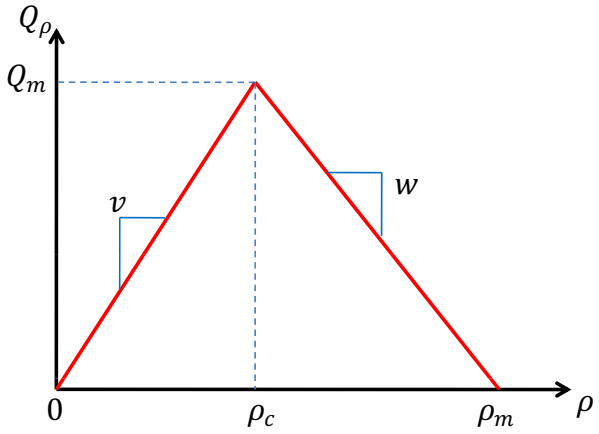

Figure 2. Fundamental Diagram

where:

s...indicates the mode ${ }^{1}$ (1:FF, 2:CC, 3:CF, 4:FC1, 5:FC2).

$\rho_{i} \ldots$ is the state $\left(\right.$ density) $=\left[\rho_{1} \ldots \rho_{5}\right]^{T}$.

$u_{i} \ldots$ is the flow input $q_{i n}$, measured on-ramp $q_{r}$, measured off-ramp $q_{s}$, and density output $\rho_{\text {out }},=\left[q_{\text {in }}, 0, q_{r}, q_{s}, \rho_{\text {out }}\right]$ $\rho_{m, i} \ldots$ is the vector of maximum (jam) density $=$ $\left[\rho_{m, 1} \ldots \rho_{m, 5}\right]^{T}$, and, see figure (2)

$Q_{m, i \cdots}$ is the vector of maximum flow rates (capacity) $\left[Q_{m, 1} \ldots Q_{m, 5}\right]^{T}$.

Here, we mention the state matrices in the FF, CC and $\mathrm{CF}$ modes, because these modes can be observed [7], [10], while the other modes (FC1, FC2) cannot be observed (see table I), therefore, we found that it is not necessary to mention them.

1) In the FF mode, the state matrices are:

$$
R_{1-5,1}=\left[\begin{array}{ccccc}
-\frac{v_{1}}{L_{1}} & 0 & 0 & 0 & 0 \\
\frac{v_{1}}{L_{2}} & -\frac{v_{2}}{L_{2}} & 0 & 0 & 0 \\
0 & \frac{v_{2}}{L_{3}} & -\frac{v_{3}}{L_{3}} & 0 & 0 \\
0 & 0 & \frac{v_{3}}{L_{4}} & -\frac{v_{4}}{L_{4}} & 0 \\
0 & 0 & 0 & \frac{v_{4}}{L_{5}} & -\frac{v_{5}}{L_{5}}
\end{array}\right]
$$

2) In the $\mathrm{CC}$ mode, the state matrices are:

${ }^{1}$ Free flow-Free flow (FF), Congested-Congested (CC), Congested-Free flow (CF), Free flow-Congested1 (CF1), Free flow-Congested2 (CF2). 
Table I

OBSERVABILITY FOR DIFFERENT SMM MODES

$$
\begin{gathered}
R_{1-5,2}=\left[\begin{array}{ccccc}
-\frac{w_{1}}{L_{1}} & \frac{w_{2}}{L_{1}} & 0 & 0 & 0 \\
0 & -\frac{w_{2}}{L_{2}} & \frac{w_{3}}{L_{2}} & 0 & 0 \\
0 & 0 & -\frac{w_{3}}{L_{3}} & \frac{w_{4}}{L_{3}} & 0 \\
0 & 0 & 0 & -\frac{w_{4}}{L_{4}} & \frac{w_{5}}{L_{4}} \\
0 & 0 & 0 & 0 & -\frac{w_{5}}{L_{5}}
\end{array}\right] \\
M_{1-5,2}=\left[\begin{array}{cccccc}
0 & 0 & 0 & 0 & 0 \\
0 & 0 & \frac{1}{L_{2}} & 0 & 0 \\
0 & 0 & 0 & 0 & 0 \\
0 & 0 & 0 & -\frac{1}{L_{4}} & 0 & \\
N_{1-6,2} & 0 & 0 & 0 & 0 & \frac{w_{6}}{L_{5}}
\end{array}\right] \\
\left.\begin{array}{ccccccc}
\frac{w_{1}}{L_{1}} & -\frac{w_{2}}{L_{1}} & 0 & 0 & 0 & 0 \\
0 & \frac{w_{2}}{L_{2}} & -\frac{w_{3}}{L_{2}} & 0 & 0 & 0 \\
0 & 0 & \frac{w_{3}}{L_{3}} & -\frac{w_{4}}{L_{3}} & 0 & 0 \\
0 & 0 & 0 & \frac{w_{4}}{L_{4}} & -\frac{w_{5}}{L_{4}} & 0 \\
0 & 0 & 0 & 0 & \frac{w_{5}}{L_{5}} & -\frac{w_{6}}{L_{5}}
\end{array}\right]
\end{gathered}
$$

3) In the $\mathrm{CF}$ mode. For the simulation, it is assumed that the wave front is located in between cells 3 and 4 and the state matrices are:

$$
\begin{aligned}
R_{1-5,3} & =\left[\begin{array}{ccccc}
-\frac{w_{1}}{L_{1}} & \frac{w_{2}}{L_{1}} & 0 & 0 & 0 \\
0 & -\frac{w_{2}}{L_{2}} & \frac{w_{3}}{L_{2}} & 0 & 0 \\
0 & 0 & -\frac{w_{3}}{L_{3}} & 0 & 0 \\
0 & 0 & 0 & -\frac{v_{4}}{L_{4}} & 0 \\
0 & 0 & 0 & \frac{v_{4}}{L_{5}} & -\frac{v_{5}}{L_{5}}
\end{array}\right] \\
M_{1-5,3} & =\left[\begin{array}{ccccc}
0 & 0 & 0 & 0 & 0 \\
0 & 0 & \frac{1}{L_{2}} & 0 & 0 \\
0 & 0 & 0 & 0 & 0 \\
0 & 0 & 0 & 0 & 0 \\
0 & 0 & 0 & -\frac{1}{L_{5}} & 0
\end{array}\right]
\end{aligned}
$$

\begin{tabular}{ccc}
\hline \hline Upstream Cells & Downstream Cells & Observable with \\
\hline Free-flow & Free-flow & Downstream meas. \\
Congested & Congested & upstream meas. \\
Congested & Free-flow & Up. \& Down. meas. \\
Free-flow & Congested 1 & Unobservable \\
Free-flow & Congested 2 & Unobservable \\
\hline
\end{tabular}

$$
\begin{gathered}
N_{1-6,3}=\left[\begin{array}{cccccc}
\frac{w_{1}}{L_{1}} & -\frac{w_{2}}{L_{1}} & 0 & 0 & 0 & 0 \\
0 & \frac{w_{2}}{L_{2}} & -\frac{w_{3}}{L_{2}} & 0 & 0 & 0 \\
0 & 0 & \frac{w_{3}}{L_{3}} & 0 & 0 & 0 \\
0 & 0 & 0 & 0 & 0 & 0 \\
0 & 0 & 0 & 0 & 0 & 0
\end{array}\right] \\
O_{1-5,3}=\left[\begin{array}{ccccc}
0 & 0 & 0 & 0 & 0 \\
0 & 0 & 0 & 0 & 0 \\
0 & 0 & 0 & -\frac{1}{L_{3}} & 0 \\
0 & 0 & 0 & \frac{1}{L_{4}} & 0 \\
0 & 0 & 0 & 0 & 0
\end{array}\right]
\end{gathered}
$$

\section{REDUCED-ORDER OBSERVER}

In this section, we will investigate how the state variable (density) of traffic flow can be estimated using a mathematical model (SMM model) and a few measurement. This is done by building an observer that uses measurements of the inputs and outputs of a linear system, along with a model of the system dynamics, to estimate the state.

The estimator design method called Full-Order Estimators reconstructs the entire state vector using measurements of some of the state variables. If sensors have no noise, then a full-order estimator contains redundancies, and it seems reasonable to question the necessity of estimating state variables that are measured directly [15].

The reduced-order observer reduces the order of the observer by the number of sensed outputs. To derive this observer, we start with the assumption that the output equals the first state as, for example $y=x_{1}$. We now partition the state vector into two parts: $x_{1}$, which is directly measured, and $x_{2}$, which represents the remaining state variables that need to be observed. If we partition the system matrices accordingly, the complete description of the system is given by:

$$
\left[\begin{array}{c}
\dot{x}_{1} \\
\dot{x}_{2}
\end{array}\right]=\left[\begin{array}{ll}
A_{11} & A_{12} \\
A_{21} & A_{22}
\end{array}\right]\left[\begin{array}{l}
x_{1} \\
x_{2}
\end{array}\right]+\left[\begin{array}{l}
B_{1} \\
B_{2}
\end{array}\right] u
$$




$$
y=\left[\begin{array}{ll}
1 & 0
\end{array}\right]\left[\begin{array}{l}
x_{1} \\
x_{2}
\end{array}\right]
$$

The dynamics of the unmeasured state variables are given by

$$
\dot{x}_{2}=A_{21} x_{1}+A_{22} x_{2}+B_{2} u
$$

where the rightmost two terms are known and can be considered as an input into the $x_{2}$ dynamics. Because $x_{1}=y$, the measured dynamics are given by the scalar equation

$$
\dot{x}_{1}=\dot{y}=A_{11} x_{1}+A_{12} x_{2}+B_{1} u
$$

If we collect the known terms of equation (17) on one side,

$$
A_{12} x_{2}=\underbrace{\dot{y}-A_{11} x_{1}-B_{1} u}_{\text {known measurement }}
$$

We obtain a relationship between known quantities on the right side, which we consider measurements, and unknown state variable on the left. Therefore, equation (17) and equation (18) have the same relationship to the state $x_{2}$ that the original equation (16) had to the entire state $\mathrm{x}$. Following this line of reasoning, we can establish the following substitutions in the original estimator equations ${ }^{2}$ to obtain a (reduced-order) observer of $x_{2}$.

$x \longleftarrow x_{2}, F \longleftarrow A_{22}, G u \longleftarrow A_{21} x_{1}+B_{2} u, y \longleftarrow$ $\dot{y}-A 11 x_{1}-B_{1} u$ and $H \longleftarrow A_{12}$

Therefore, the reduced-order observer equation obtained by substituting these items in equation (19)

$\dot{\hat{x}}_{2}=A_{22} \hat{x}_{2}+\underbrace{A_{21} x_{1}+B_{2} u}_{\text {input }}+L(A_{12} \hat{x}_{2}-\underbrace{\dot{y}+A_{11} x_{1}+B_{1} u}_{\text {measurement }})$

If we define the estimator error to be $\tilde{x}_{2} \triangleq \hat{x}_{2}-x_{2}$. Then the dynamics of the error are given by subtracting equation (16) from equation (20) to get

$$
\dot{\tilde{x}}_{2}=\left(A_{22}+L A_{12}\right) \tilde{x}_{2}
$$

and its characteristic equation of the error is given by

$$
\operatorname{det}\left[s I-\left(A_{22}+L A_{12}\right)\right]=0
$$

$\mathrm{L}$ is a matrix that tells how the error $\tilde{x}_{2}$ is weighted and distributed among the states. If we choose $\mathrm{L}$ so that $\left(A_{22}+L A_{12}\right)$ has stable and reasonably fast eigenvalues, then the error will decay to zero. This means that $\hat{x}_{2}$ will converge to $x_{2}$. Now equation (20) can be rewritten as

$\dot{\hat{x}}_{2}=\left(A_{22}+L A_{12}\right) \hat{x}_{2}+\left(A_{21}+L A_{11}\right) x_{1}+\left(B_{2}+L B_{1}\right) u-L \dot{y}$

The fact that we must form the derivative of the measurements $(\dot{y})$ in equation (23) appears to present a practical difficulty. It

\footnotetext{
${ }^{2}$ The original estimator equations means the golden rule [15]. When in trouble, use feedback. Consider feeding back the difference between the estimated and measured outputs and correcting the model continuously with this error signal. The equation for this scheme is:

$$
\dot{\hat{x}}=F \hat{x}+G u+L(H \hat{x}-y)
$$

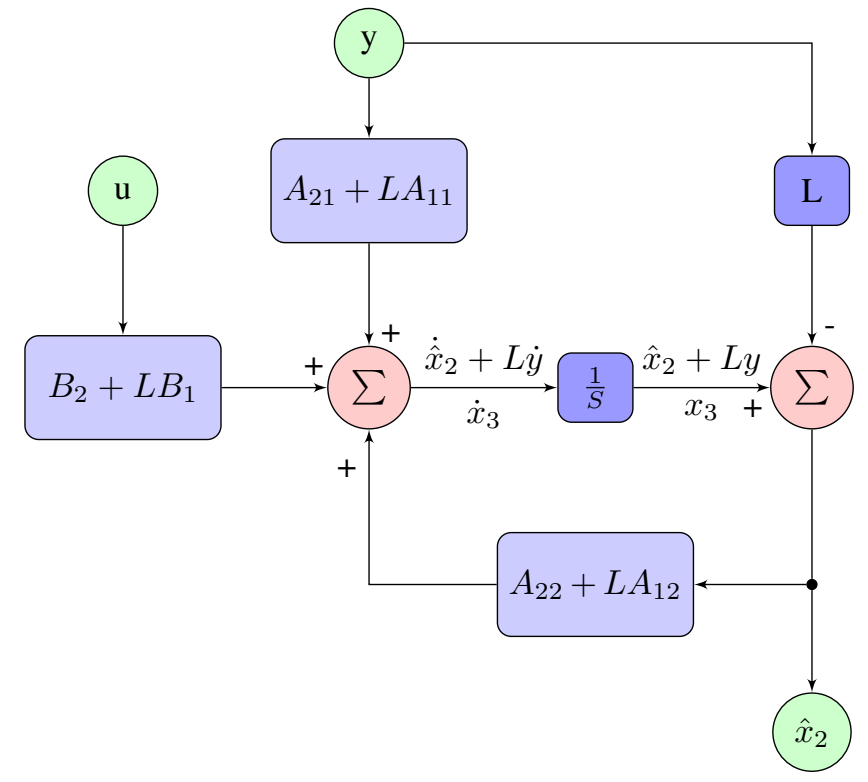

Figure 3. flow chart (Reduced-order estimator)

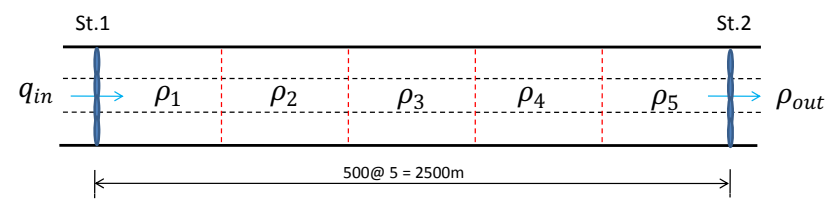

Figure 4. Simulated Road Section

is known that differentiation amplifies noise, so if $(y)$ is noisy, the use of $(\dot{y})$ is unacceptable. To get around this difficulty, we define the new controller state to be:

$$
x_{3} \triangleq \hat{x}_{2}+L y
$$

In terms of this new state the implementation of the reducedorder observer is given by:

$$
\begin{aligned}
& \dot{x}_{3}=\dot{\hat{x}}_{2}+L \dot{y}= \\
& \left(A_{22}+L A_{12}\right) \hat{x}_{2}+\left(A_{21}+L A_{11}\right) x_{1}+\left(B_{2}+L B_{1}\right) u
\end{aligned}
$$

and $\dot{y}$ no appears directly. A block diagram representation of the reduced-order estimator is shown in figure 3 .

\section{Simulation And Results}

Figure 4 is a schematic diagram of the freeway portion used in the simulation. It shows the mainline segment partitioned into 5 identical cells, $2.5 \mathrm{~km}$ in length (each cell length = 500 meters). Two mainline loop detector (sensor) stations are labeled (St.1) and (St.2).

The traffic model (SMM) is a hybrid model with 5 different modes. At any time, the system can change from one mode to another. In order to the system functions permanently, the observer is designed and implemented for the modes FF and $\mathrm{CC}$, that have been simulated. For the case of $\mathrm{CF}$ mode, it is assumed that there is a wave front between cell 3 and Cell 4. It means that the capacity at between cells 3 and 4 is 
reduced. Thus, the traffic cannot flow with maximum capacity. In consequence, congestion is produced from cell 3 to the upstream, and traffic situation will be free flow from cell 4 to the downstream.

1) In the case of FF mode, the equations of state variable (density) are implemented as follows:

$$
\left[\begin{array}{c}
\dot{\hat{\rho}}_{1} \\
\dot{\hat{\rho}}_{2} \\
\dot{\hat{\rho}}_{3} \\
\dot{\hat{\rho}}_{4} \\
\dot{\hat{\rho}}_{5}
\end{array}\right]=\left[\begin{array}{c}
\left(R_{11}+L R_{21}\right) \hat{\rho}_{1}+L R_{22} \rho_{2}+M_{11} q_{i n}-L \dot{\rho}_{2} \\
\left(R_{22}+L R_{32}\right) \hat{\rho}_{2}+R_{21} \rho_{1}+L R_{33} \rho_{3}-L \dot{\rho}_{3} \\
\left(R_{33}+L R_{43}\right) \hat{\rho}_{3}+R_{32} \rho_{2}+L R_{44} \rho_{4}-L \dot{\rho}_{4} \\
\left(R_{44}+L R_{54}\right) \hat{\rho}_{4}+R_{43} \rho_{3}+L R_{55} \rho_{5}-L \dot{\rho}_{5} \\
R_{54} \hat{\rho}_{4}+R_{55} \hat{\rho}_{5}
\end{array}\right]
$$

2) In the case of $\mathrm{CC}$ mode, the equations of state variable (density) are implemented as follows:

$$
\left[\begin{array}{c}
\dot{\hat{\rho}}_{1} \\
\dot{\hat{\rho}}_{2} \\
\dot{\hat{\rho}}_{3} \\
\dot{\hat{\rho}}_{4} \\
\dot{\hat{\rho}}_{5}
\end{array}\right]=\left[\begin{array}{c}
R_{11} \hat{\rho}_{1}+R_{12} \hat{\rho}_{2}+N_{11} \rho_{m 1}+N_{12} \rho_{m 2} \\
\left(R_{22}+L R_{12}\right) \hat{\rho}_{2}+R_{23} \rho_{3}+L R_{11} \rho_{1}+N_{22} \rho_{m 2}+ \\
N_{23} \rho_{m 3}+L\left(N_{11} \rho_{m 1}+N_{12} \rho_{m 2}\right)-L \dot{\rho}_{1} \\
\left(R_{33}+L R_{23}\right) \hat{\rho}_{3}+R_{34} \rho_{4}+L R_{22} \rho_{2}+N_{33} \rho_{m 3}+ \\
N_{34} \rho_{m 4}+L\left(N_{22} \rho_{m 2}+N_{23} \rho_{m 3}\right)-L \dot{\rho}_{2} \\
\left(R_{44}+L R_{34}\right) \hat{\rho}_{4}+R_{45} \rho_{5}+L R_{33} \rho_{3}+N_{44} \rho_{m 4} \\
+N_{45} \rho_{m 5}+L\left(N_{33} \rho_{m 3}+N_{34} \rho_{m 3}\right)-L \dot{\rho}_{3} \\
\left(R_{55}+L R_{45}\right) \hat{\rho}_{5}+L R_{44} \rho_{4}+M_{55} \rho_{\text {out }}+N_{55} \rho_{m 5} \\
+N_{56} \rho_{m, \text { out }}+L N_{44} \rho_{m 4}+L N_{45} \rho_{m 5}-L \dot{\rho}_{4}
\end{array}\right]
$$

3) In the case of CF mode, the equations of state variable (density) are implemented as follows:

$$
\left[\begin{array}{c}
\dot{\hat{\rho}}_{1} \\
\dot{\hat{\rho}}_{2} \\
\dot{\hat{\rho}}_{3} \\
\dot{\hat{\rho}}_{4} \\
\dot{\hat{\rho}}_{5}
\end{array}\right]=\left[\begin{array}{c}
R_{11} \hat{\rho}_{1}+R_{12} \hat{\rho}_{2}+N_{11} \rho_{m 1}+N_{12} \rho_{m 2} \\
\left(R_{22}+L R_{12}\right) \hat{\rho}_{2}+R_{23} \rho_{3}+L R_{11} \rho_{1}+N_{22} \rho_{m 2}+ \\
N_{23} \rho_{m 3}+L\left(N_{11} \rho_{m 1}+N_{12} \rho_{m 2}\right)-L \dot{\rho}_{1} \\
\left(R_{33}+L R_{23}\right) \hat{\rho}_{3}+L R_{22} \rho_{2}+N_{33} \rho_{m 3} \\
+O_{34} q_{m 4}+L\left(N_{22} \rho_{m 2}+N_{23} \rho_{m 3}\right)-L \dot{\rho}_{2} \\
\left(R_{44}+L R_{54}\right) \hat{\rho}_{4}+L R_{55} \rho_{5}+O_{44} q_{m 4}-L \dot{\rho}_{5} \\
R_{55} \hat{\rho}_{5}+R_{54} \hat{\rho}_{4}
\end{array}\right]
$$

The theoretical results are illustrated by some simulations. The maximum (jam) density $\rho_{m}=180 \mathrm{veh} / \mathrm{km}$, the free flow speed $v_{i}=90 \mathrm{~km} / \mathrm{h}$ and the congestion speed $w_{i}=$ $22.5 \mathrm{~km} / \mathrm{h}$. The $\mathrm{L}$ values for $\mathrm{FF}, \mathrm{CC}$ and $\mathrm{CF}$ modes respectively are $1 / 5,-1$ and 1 . The macroscopic simulation has been done during 100 minutes. In order to produce different modes of the SMM model, several types of data are used, one for producing the mode FF, one for producing CC and the other for producing CF. So the upstream flow $q_{i n}$ and the downstream density $\rho_{\text {out }}$ of the main line are given a way that the system can produce these three different modes (see figure 5). As we can realize (see figure 5-top), the flow used at the upstream, is always inferior to the capacity in the case of FF mode. While, the flow is superior or equal to capacity
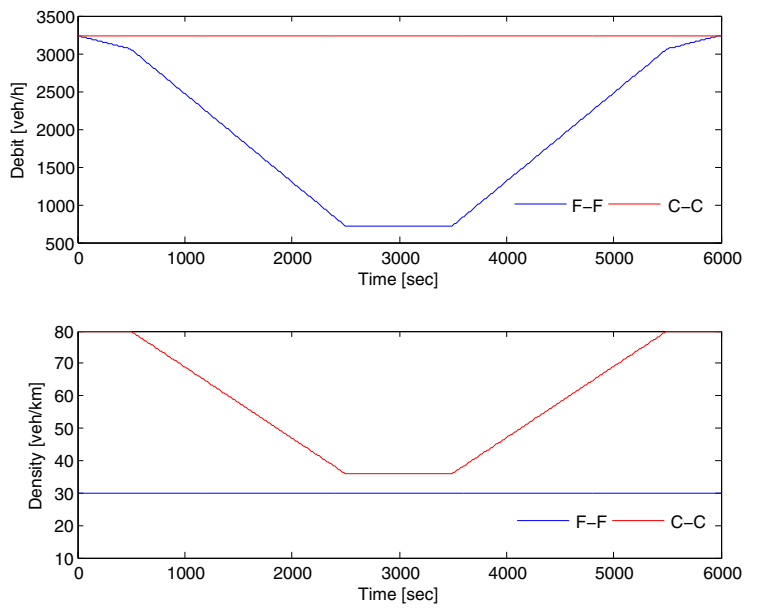

Figure 5. Debit of upstream and density of downstream of the boundary line

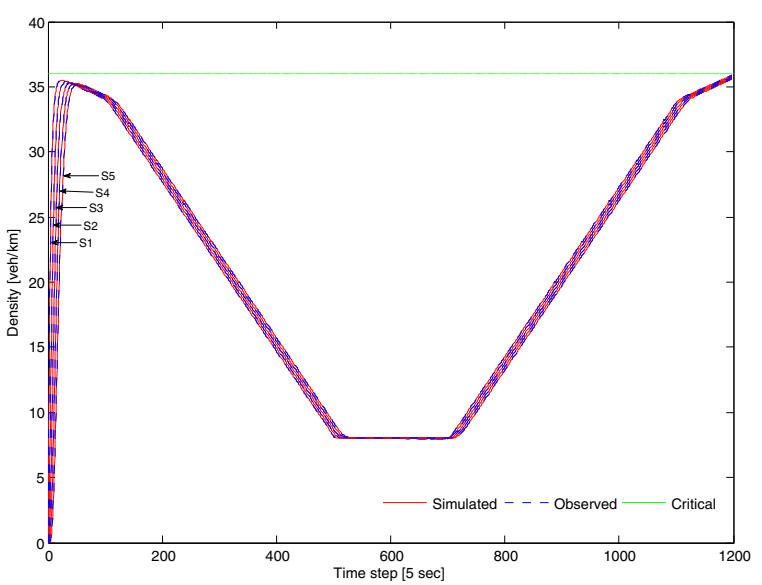

Figure 6. Simulated and observed density for all the 5 cells in the FF mode

in the case of CC and CF modes. However, the density used at the downstream of the mainline is given in figure 5-bottom, it is obvious that the density for FF and CF modes is inferior the critical density. While the density in the CC mode is always superior or equal to critical density.

In figure 6 , results of the FF mode is shown, it can be noted that the observer reproduce the same trajectory as the SMM model for all the cells. Figures 7, 8 and 9 show the same results for the $\mathrm{CC}$ and $\mathrm{CF}$ modes respectively. The simulation with noisy data (Mean $=0$ and variance $=0.0016$ ) is also tested, it is shown in figure 10, and 11 for the FF and $\mathrm{CC}$ mode respectively.

\section{CONCLUSiON}

In this paper, we have demonstrated the relevance of reduced-order observer introduced by Luenberger in the intelligent transportation systems area and particularly in freeway traffic flow domain. The used macroscopic model rests on the so-called SMM, which is linear time-varying model. Several numerical simulations conducted for different modes of the 


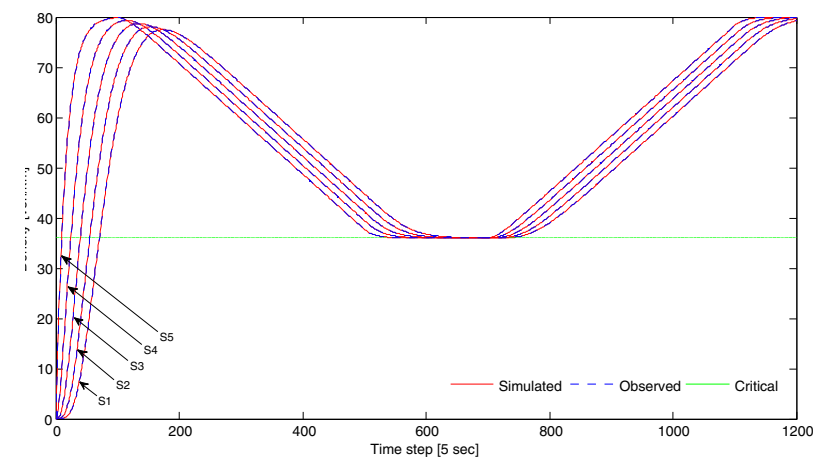

Figure 7. Simulated and observed density for all the 5 cells in the CC mode

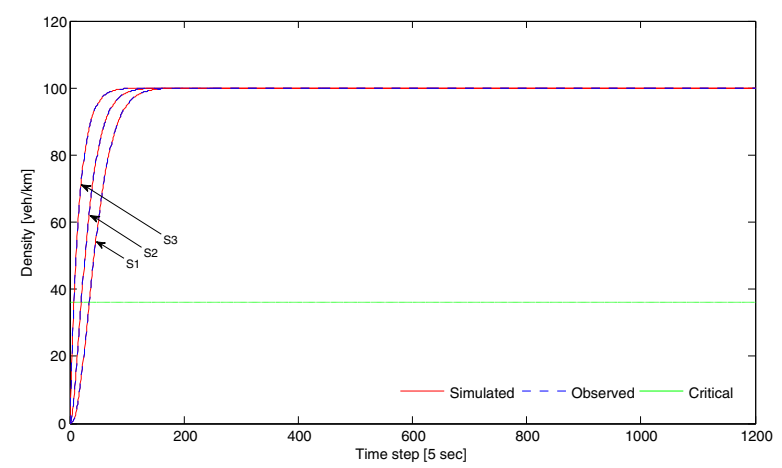

Figure 8. Simulated and observed density for all the upstream cells of the wave front in the CF mode

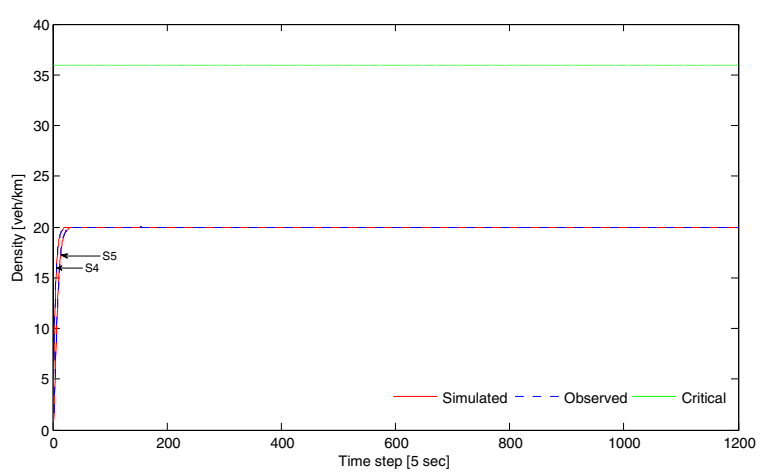

Figure 9. Simulated and observed density for all the downstream cells of the wave front in the $\mathrm{CF}$ mode

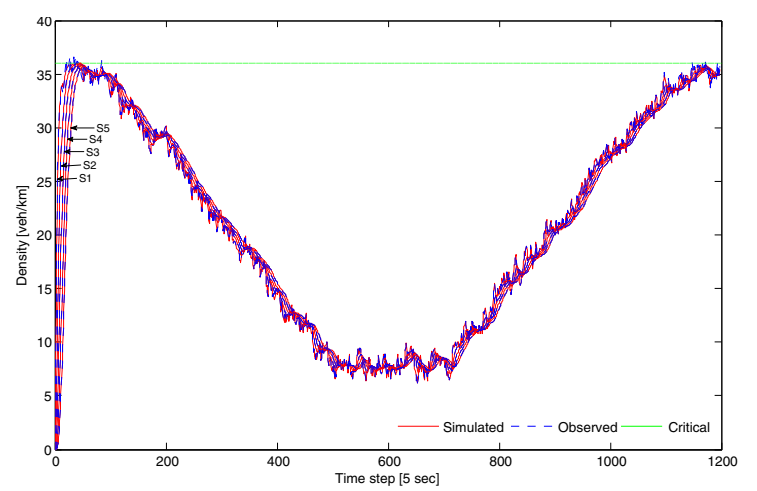

Figure 10. Simulated and observed density for all the 5 cells in the FF mode with noisy data

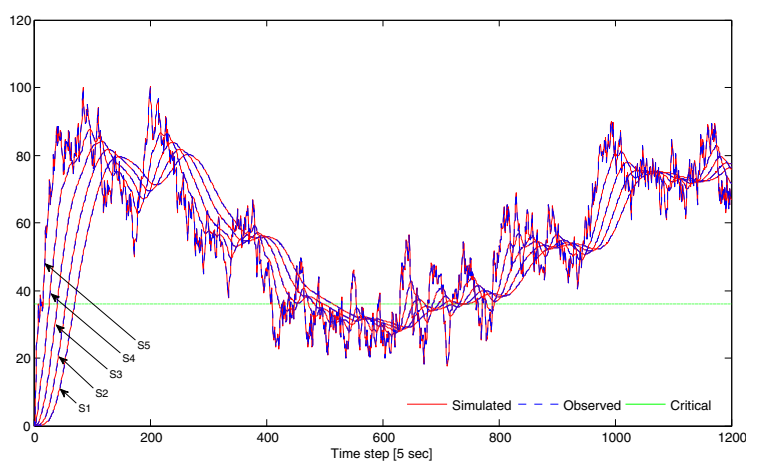

Figure 11. Simulated and observed density for all the 5 cells in the CC mode with noisy data

traffic, show robust and efficient results even with noisy data. Further works will consider this identification method for more complex system including field real data, and will introduce some control algorithm for ramp metering in order to alleviate the congestion phenomena.

\section{REFERENCES}

[1] David G. Luenberger. An introduction to observers. IEEE Transactions on Automatic Control, vol. AC-16, NO.6:Pages: 596-602, 1971.

[2] N. E. Nahi. Freeway traffic data processing. IEEE, vol. 61:Pages: 537$541,1973$.

[3] D. C. Gazis and C. H. Knapp. On-line estimation of traffic densities from time-series of flow and speed data. Transportation science, vol. 5:pages: 283-302, 1971.

[4] C. H. Knapp. Traffic estimation and density estimation for single and multi-lane traffic. Transportation Science, vol. No.7:pages: 75-84, 1973.

[5] M. Papageorgiou. Application of automatic control concepts to traffic flow modeling and control. Springer-Verlag, vol. 50, 1983.

[6] Rashid R. Kohan. Robust State Estimation and Control of Highway Traffic Systems. PhD thesis, Electrical and Computer Engineering/ University of Toronto, 2001.

[7] Laura Muñoz Xiaotian Sun Roberto Horowitz Luis Alvarez. Traffic density estimation with the cell transmission model. IEEE, pages pages: 3750-3755, 2003.

[8] Xiaotian Sun Laura Muñoz and Roberto Horowitz. Mixture kalman filter based highway congestion mode and vehicle density estimator and its application. American Control Conference, pages pages: 2098-2103, 2004.

[9] K. Stan̆ková and B. De Schutter. On freeway traffic density estimation for a jump markov linear model based on daganzo's cell transmission model. In Proceedings of the 13th International IEEE Conference on Intelligent Transportation Systems (ITSC 2010), Madeira Island, Portugal, PP. 13-18, 2010.

[10] Irinel-Constantin Morărescu and Carlos Canudas de Wit. Highway traffic model-based density estimation. In American Control ConferenceACC, 2011.

[11] M. J. Lighthill and G.B. Whitham. On kinematic waves ii: A theory of traffic flow in long crowded roads. Proc. Royal Soc. London, vol. A-229:Pages: 317-345, 1955.

[12] P.I. Richards. Shock waves on the highway. Operations Research, vol.4:Pages: 42-51, 1956.

[13] Carlos F. Daganzo. The cell transmission model: A dynamic representation of highway traffic consistent with the hydrohynamic theory. Transportation Research-B, vol. 28(4):pages: 269-287, 1994.

[14] Carlos F. Daganzo. The cell transmission model, part ii: Network traffic. Transportation Research-B, vol. 29(2):pages: 79-93, 1995.

[15] Gene F. Franklin J. David Powell Abbas Emami-Naeini. Feedback Control of Dynamic Systems. 2002. 